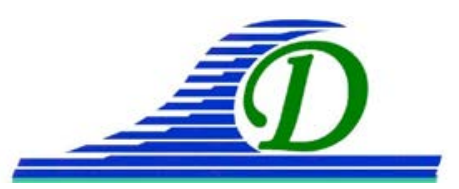

XIII ${ }^{\text {èmes }}$ Journées Nationales Génie Côtier - Génie Civil Dunkerque, 2-4 juillet 2014

DOI:10.5150/jngcgc.2014.076 ～(C) Editions Paralia CFL

disponible en ligne - http://www.paralia.fr - available online

\title{
Port-La Nouvelle. Etapes du débat public sur le projet d'extension de l’infrastructure portuaire
}

\author{
Stéphane SCARDIGLI ${ }^{1}$, Arnaud BRASSEUR ${ }^{2}$
}

1. OCEANIDE, Assistant Maître d’Ouvrage, Port de Brégaillon, BP 63, 83502 France. sscardigli@oceanide.net

2. Chef de projet "Grand Port de PLN", Conseil Région Languedoc Roussillon, Direction des Transports et des Communications, Sous-Direction des Ports, Pôle Maîtrise d’Ouvrage, Montpellier, France.

Brasseur.Arnaud@cr-languedocroussillon.fr

\section{Résumé :}

La Région Languedoc-Roussillon a saisi la Commission Nationale du Débat Public (CNDP) en septembre 2011 pour un projet d'extension portuaire d'environ 200 millions d'euros, donc supérieur au seuil de 150 millions d'euros au-dessus duquel, pour un tel projet, le code de l'environnement (articles L121-1 à L121-15 et R121-1 à R121-16) rend la saisine de la CNDP obligatoire. La CNDP a décidé le 9 novembre 2011 que le projet devait faire l'objet d'un débat public que la Commission organisera elle-même et dont elle confiera l'animation à une commission particulière (CPDP). Le président de la CPDP a été nommé le 7 décembre 2011 puis, compte tenu de la période électorale, l'équipe de la Commission Particulière fut constituée en juin 2012. Quelques mois de travail avec la CPDP ont ensuite été nécessaires avant le début du débat, notamment afin d'élaborer les différents supports du débat (dont le dossier du maître d'ouvrage). Le débat s’est tenu du 17 décembre 2012 au 16 avril 2013.

Le débat public est une étape réglementaire fondamentale pour un projet d'une telle ampleur. En effet, un avis peu favorable de la commission, un débat trop politisé ou mal préparé peut conduire à l'arrêt du projet, comme ce fut le cas pour le projet d'extension du port de Nice (débat public du 15 octobre 2001 au 31 janvier 2002).

Le présent papier vise à décrire les principales étapes d'un projet de création ou d'extension d'une infrastructure portuaire depuis sa préparation, la rédaction du dossier du maître d'ouvrage, les réunions publiques, jusqu'à l'avis de la commission nationale de débat public. Sont présentés en particulier les temps forts du débat (rédaction du dossier du maitre d'ouvrage, réunions techniques, points importants, ...), ainsi que quelques aspects qui peuvent contribuer à son succès (étude de faisabilité et préparation du projet, réponses aux questions en cours de débat, sujets à aborder, ...).

Mots-clés : Projet Grand Port, Travaux maritimes, Réglementation, Débat Public. 


\section{Thème 4 - Ouvrages portuaires et offshore}

\section{Introduction}

Pour un projet de création ou d'extension d'une infrastructure portuaire, l'étape du débat public reste un moment clé. Elle est le fruit d'un travail préparatoire qui passe notamment par la réalisation d'études de faisabilité puis la rédaction du dossier du maître d'ouvrage (DMO).

Pour le projet d'extension de l'infrastructure portuaire de Port-La Nouvelle (Aude), ce sont ensuite 10 réunions qui ont ponctué les échanges avec des présentations du projet, des réunions d'expression et un atelier technique ad hoc.

\section{Etude de faisabilité}

\subsection{Les projets}

Le port de Port-La Nouvelle est spécialisé dans la réception des hydrocarbures ( $2{ }^{\text {ème }}$ port français de Méditerranée) et dans l'exportation des céréales ( $1^{\mathrm{er}}$ port méditerranéen). Le port est actuellement en limite de capacité et ne peut envisager aucune augmentation de son activité.

Transféré par l'Etat à la Région Languedoc-Roussillon en janvier 2007, cette dernière a alors engagé des études de faisabilité qui ont été menées par OCEANIDE (2009-2012). Elles ont principalement consisté à définir trois grands schémas d'aménagements : amélioration du port (pas de nouveaux ouvrages maritimes), réaménagement de l'avantport et de la passe d'entrée (construction de deux kilomètres de digue), agrandissement du port (extension du port avec trois kilomètres de nouvelles digues).

Le maître d’ouvrage a proposé au débat ces trois scénarios du port. La figure suivante les présente.
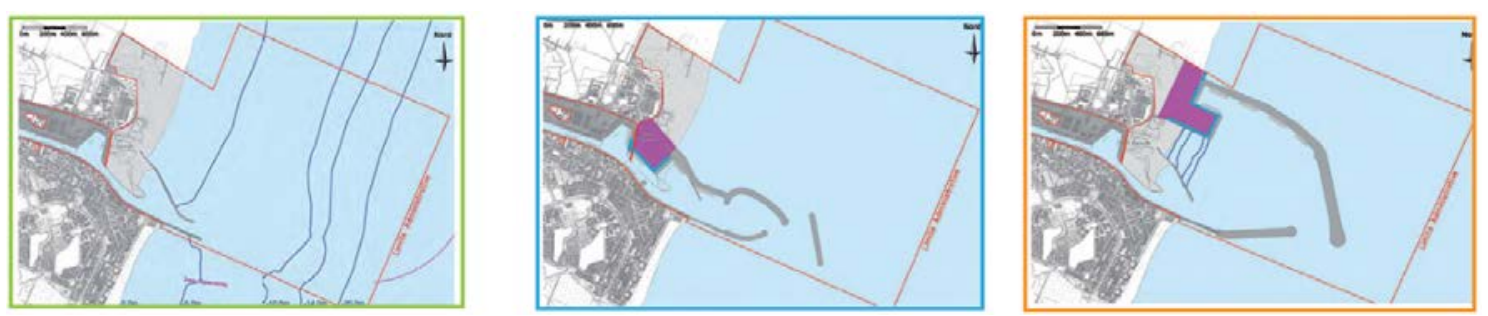

Figure 1. Scénarios d'aménagement du port de Port-La Nouvelle

\subsection{Les autres actions préparatoires}

Outre l'étude de faisabilité qui a permis un premier tracé des ouvrages, l'étude et l'évaluation de plusieurs scénarios, citons quelques études importantes menées avant l'ouverture du débat :

a) Etudes socio-économiques (AMO, 2012)

b) Etude matériaux (AMO, 2012)

c) Diagnostic environnemental du milieu maritime (Safege, 2012) 


\section{XIII ${ }^{\text {èmes }}$ Journées Nationales Génie Côtier - Génie Civil \\ Dunkerque, 2-4 juillet 2014}

\section{Le dossier du maître d'ouvrage}

Le DMO est constitué d'une centaine de pages (hors annexes). Il est rédigé par le maître d'ouvrage et ses assistants, à partir des recommandations de la CPDP.

Environ 6 mois ont été nécessaires à la finalisation de cette pièce maîtresse du débat public.

\subsection{Les chapitres}

I Port-La nouvelle au cœur du développement social et économique de la Région Languedoc-Roussillon

I-1 Le contexte du transport maritime

I-2 Le contexte portuaire

I-3 L'économie logistique régionale

II Le projet d'agrandissement du port de Port-La Nouvelle

II-1 Les caractéristiques actuelles du port de commerce de Port-La Nouvelle

II-2 Les options du projet maritime

II-3 Le projet d'agrandissement du port

II-4 L’approvisionnement en matériaux du chantier

III Les ambitions socio-économiques du projet

III-1 Impulser un nouveau démarrage économique

III-2 Bénéficier de l’attractivité de la région

III-3 Activités présentes et perspectives des principales filières économiques du port

III-4 Activités du port de pêche et de plaisance

III-5 Les enjeux socio-économiques du projet de Port-La Nouvelle

III-6 Les conséquences d'un statut quo

IV Insertion du projet dans son territoire

IV-1 Les enjeux urbains dans la relation ville-port

IV-2 Un environnement à préserver et à valoriser

IV-3 Les interactions du projet avec l'environnement

V Après le débat public

\subsection{Quelques points importants}

Un aménagement ou une extension portuaire doit être conçue avec l'aménagement d'une chaîne logistique terrestre en lien avec les nouvelles capacités et en synergie avec l'existant. La notion d'hinterland est donc à bien présenter avec en particulier les modes de communication (liaisons routières, aéroportuaires, ferroviaires et fluviales). Pour Port-La Nouvelle, la présence de deux ports maritimes voisins (Sète et Port-Vendres), dans un périmètre géographique relativement réduit, aurait pu faire émerger la crainte d'une concurrence entre les ports. La complémentarité entre les ports, l'étude des 


\section{Thème 4 - Ouvrages portuaires et offshore}

synergies possibles et la mise en place d'une gouvernance régionale ont donc été privilégiées et mises en avant.

Les atouts du projet ne sont évidemment pas à négliger et sont à souligner : par exemple, la réserve foncière de plus de 100 hectares du projet de Port-La Nouvelle est un atout non négligeable peu répandu, idéal pour la mise en œuvre d'une chaîne logistique, d'espaces de stockage et indispensable à un développement industriel.

Les perspectives socio-économiques sont également un aspect fondamental. En effet, des nombreuses discussions ont porté sur les trafics existants et futurs, la rentabilité du nouveau port, les emplois directs, indirects ou induits.

La taille des bateaux susceptibles de venir dans un port est liée à l'évolution des trafics. D'un point de vue technique, ce sont d'ailleurs les caractéristiques des navires projets qui dictent le tracé des ouvrages portuaires. Même s'il est difficile dans un secteur fluctuant où la visibilité est assez réduite de se livrer à un exercice de prévision sur le long terme, un nouveau port doit voir sur le long terme, anticiper et proposer de nouvelles opportunités. Ce message doit être longuement préparé et argumenté afin d'être bien partagé.

Le port actuel de Port-La Nouvelle est actuellement très proche de la ville (certains parlent de "port dans la ville"). Les plus gros pétroliers sont actuellement déchargés en mer via un sea-line. L’aspect sécurité et les améliorations apportées par un réaménagement doivent bien être mis en avant. Le Plan de Prévention des Risques Technologiques, en cours d'élaboration est d'ailleurs un élément que le projet doit prendre en compte.

Un développement portuaire n'est pas forcément en contradiction avec la préservation de l'environnement. Même si un tel projet nécessite après le débat de nombreuses investigations, études (étude d'impact) et concertations (enquête publique), il n'en reste pas moins qu'une bonne connaissance de l'état actuel, une démarche de développement durable, une première évaluation des effets pressentis (impacts positifs et négatifs, directs et indirects) et la proposition de solutions de réduction voir compensatoires doivent être clairement présentées dès le stade du DMO.

\section{Les réunions}

Le nombre de réunions est en général déterminé par la CPDP en fonction des enjeux du projet et du territoire concerné. Pour Port-La Nouvelle, 10 réunions ont été effectuées dans diverses communes de la région (Port-La Nouvelle, Montpellier, Sète, Narbonne, Port-Vendres, ...). Les Nouvellois auraient souhaité plus de réunions dans leur commune (seule une réunion y a eu lieu).

On remarquera les horaires tardifs des réunions (début à 19h00) et leur longueur (entre $3 \mathrm{~h} 30$ et $4 \mathrm{~h} 30$ ). 


\section{XIII ${ }^{\text {èmes }}$ Journées Nationales Génie Côtier - Génie Civil \\ Dunkerque, 2-4 juillet 2014}

\subsection{Les réunions de présentation}

Quatre réunions thématiques correspondant à la présentation des quatre chapitres constituant le DMO ont eu lieu.

Une cinquième réunion a permis une présentation de la synthèse du débat.

\subsection{Les réunions d'expression}

56 cahiers d'acteurs (document de 5 pages) ont été rédigés (associations, CCI, acteurs locaux, syndicats...), suscités par les contacts pris par la CPDP, ce qui témoigne que le projet suscitait de nombreuses réactions. Quatre réunions relatives à leur présentation ont été nécessaires.

\subsection{Atelier ad hoc}

Cet atelier a permis des échanges techniques entre trois experts nommés par la CPDP et trois représentants du maître d'ouvrage.

Les conclusions de ses travaux ont été présentées en séance publique, et la compétence des experts venus s'exprimer a grandement facilité la compréhension du public concernant les enjeux du projet, tant sur le plan technique que sur celui des conséquences économiques.

\section{La conduite du débat et son déroulement}

\subsection{Les participants}

M. Pierre-Frédéric TENIERE-BUCHOT a été le président de la Commission Particulière du Débat Public. 4 autres membres ont constitué cette commission (Etienne Ballan, Patricia Brossier, François Perdrizet et Camille Saïsset).

Le maître d'ouvrage a été directement représenté lors de deux réunions par son Président (Christian Bourquin) et pour toutes les réunions par son premier Viceprésident (Robert Navarro), en charge des transports et de l'intermodalité. Les responsables des services de la Région ont d'autre part assuré les exposés de présentation du projet, avec des représentants de l'AMO dont le maître d'ouvrage s'est entouré (Catram, Intervia, Océanide et d'une société de communication: Com.une.exception) et avec des experts reconnus (exemple: M. Chapon, ancien directeur des ports maritimes de France, M. Latteux, expert en hydro-sédimentaire). 


\section{Thème 4 - Ouvrages portuaires et offshore}

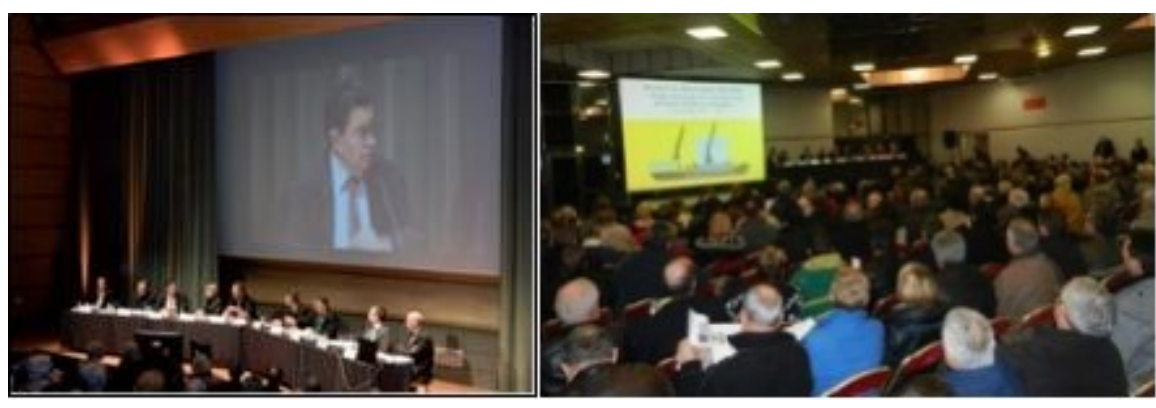

Photo 1. Le débat.

Hormis à la première réunion tenue à Port-La nouvelle, (plus de 330 participants), le grand public n’a pas fréquenté massivement les réunions (environ 150 personnes à chaque réunion). Les réunions ont essentiellement attiré des élus locaux, des membres d'associations ou de partis politiques et des acteurs du trafic portuaire.

Remarque : des entreprises externes sont également intervenues pour l'organisation du débat (secrétariat général, relation presse, supports de communication, logistique, retranscription).

\subsection{Le déroulement}

Le débat public a pour ambition d'articuler la démocratie représentative et la démocratie participative. Tous les documents sont accessibles, les échanges sont enregistrés, oralement et par écrit (site dédié : www.debatpublic-pln2015.org).

Ce débat public s'est déroulé convenablement au cours des réunions, les arguments échangés ont été de bonne qualité en général. Il a été marqué par un fort appui des politiques (projet approuvé à l'unanimité par le Conseil Régional).

La culture du débat public reste encore à s'implanter en tant qu'apport positif à la décision politique démocratique et non comme contrainte venant la limiter: des divergences peuvent apparaître entre la CPDP et le Maître d’Ouvrage.

D'un point de vue technique, on peut regretter une certaine politisation des échanges voir une confrontation politique, au détriment de l'objectif de l'enrichissement technique du projet à travers des échanges constructifs, neutres et non orientés. Ainsi, très vite, les réunions ont vu se produire le dialogue des mêmes intervenants se répondant les uns aux autres, avançant les mêmes arguments, et reprenant d'un lieu à l'autre le discours qu'ils avaient déjà tenu les semaines précédentes.

Enfin, une moindre réserve des industriels aurait certainement permis de limiter les diverses prospectives économiques et rassurer les plus réfractaires.

\section{$5.3 \underline{\text { Les avis }}$}

Tous s'accordent à dire que si rien n'est fait, le port risque de péricliter. De plus, le constat selon lequel le marché portuaire international va vers une augmentation constante de la taille des navires est également unanimement reconnu. 


\section{XIII ${ }^{\text {èmes }}$ Journées Nationales Génie Côtier - Génie Civil \\ Dunkerque, 2-4 juillet 2014}

Les acteurs favorables à l'extension sont principalement : la Chambre de Commerce et d'Industrie, les institutionnels de la Région, les opérateurs portuaires, les industriels, les syndicats, les professionnels du transport maritime.

Les habitants de Port-la Nouvelle ont également globalement accueilli le projet favorablement.

Les principaux désaccords sont apparus sur la dimension de l'infrastructure en jeu dans les scénarios “'Grand Port”. D'ailleurs, à la demande d'Europe Ecologie Les Verts et de l'association ECCLA, une expertise a alors été menée (atelier ad hoc) afin d'examiner les possibilités techniques et économiques d'un phasage des travaux envisagés pour l'extension de l'infrastructure portuaire.

C'est d'une partie du monde agricole qu'est venue l'opposition la plus marquée. En effet, la Confédération paysanne considère que le développement des capacités d'import/export agroalimentaire n'est pas souhaitable, en cela qu'elle ne créera pas d'emplois localement et qu'elle contribuera à affaiblir les agriculteurs français, mis en concurrence avec les agricultures du bassin méditerranéen.

Enfin des discussions concernant le patrimoine naturel et sauvage, la plage au Nord du port, ont eu lieu et même divisé l'assemblée entre d'un côté les Nouvellois et les amateurs de glisse qui veulent continuer à bénéficier d'un usage illégal à la plage en y circulant avec des engins motorisés, et de l'autre côté les services de l'Etat et les associations d'écologistes qui prônent le respect de la loi. Toutefois, pour la plupart des participants au débat public, le projet est apparu compatible avec la préservation de l'environnement.

\subsection{Les autres échanges}

La couverture par la presse locale a été régulière tout le long du débat (191 articles et 31 “unes” entre novembre 2012 et avril 2013).

La CPDP a rencontré le plus grand nombre possible d'acteurs (services de l'Etat, collectivités territoriales, chambres consulaires, entreprises, personnels et acteurs portuaires, associations, presse, etc.).

Au-delà des enregistrements vidéographiques et des verbatim saisis en salle, un site de questions-réponses (environ 130 au final) a permis de compléter et développer par écrit les arguments échangés entre acteurs et maître d'ouvrage.

Un film promotionnel de 6 minutes de présentation des arguments du projet fut projeté à l'accueil des séances.

\section{Conclusion}

La chronologie a été la suivante :

2009-2012 : Etude de faisabilité - études complémentaires

8 septembre 2011 : saisine de la CNDP

9 novembre 2011 : décision de la CNDP d'organiser le débat public 


\section{Thème 4 - Ouvrages portuaires et offshore}

7 décembre 2011 : nomination du président de la CPDP

Avril - juin 2012 : élection présidentielle puis législative

3 mai 2012 : prolongement de 6 mois du délai de constitution du dossier du débat

6 juin 2012 : nomination des membres de la CPDP

27 septembre 2012 : première version du DMO

7 novembre 2012 : commentaires de la CNDP sur le DMO préliminaire

5 décembre 2012 : validation du DMO

17 décembre 2012 - 16 avril 2013 : débat public

Juin 2013 : Bilan du débat public dressé par le président de la CNDP

19 juillet 2013 : Décision de la région de poursuivre et précisions sur le projet mené

Le coût imputable à la région Languedoc Roussillon du débat a été de l'ordre de 635000 euros pour les seuls frais liés à l’organisation du débat (secrétariat général, logistique des réunions, impression des supports du débat, relations presse, compte-rendu des réunions) et de 370000 euros pour les prestations de l'AMO (rédaction des supports liés au débat (DMO, présentation, film, ...) et assistance technique pour l'organisation et lors des réunions).

Les apports d'un tel débat sont difficiles à être évalués. Sans aucun doute, derrière la confrontation politique, le particulier ou l'industriel a une chance unique de participer, s'interroger voir même donner son avis. Ainsi il pourra être en attente de réponses précises, qui relèvent des études à venir (ex. l'étude d’impact). Parfois perçu comme une "contrainte administrative", il permet toutefois au Maître d'Ouvrage de mettre en place son projet et déjà de le voir analysé, plus ou moins techniquement.

\section{Glossaire}

AMO : Assistance Maitrise d’Ouvrage (représenté par le groupement Catram, Intervia, Océanide et Com.une.exception)

CNDP : Commission Nationale du Débat Public, autorité administrative indépendante

CPDP : Commission Particulière du Débat Public, nommé par la CNDP

DMO : Dossier du Maître d’Ouvrage

ECCLA : Ecologie du Carcassonnais, des Corbières et du Littoral Audois

EELV : Europe Ecologie Les Verts

PPRT : Plan de Prévention des Risques Technologiques

\section{Références bibliographiques}

Région Languedoc Roussillon (2012). Dossier du maître d'ouvrage, Débat public décembre 2012-avril 2013 sur le projet d'extension de l'infrastructure portuaire de Port-La Nouvelle.

CNDP (16 juin 2013). Port-La Nouvelle 2015, Débat public sur le projet d'extension de l'infrastructure portuaire, Compte rendu du débat public.

CNDP (juin 2013). Bilan du débat public, Projet d'extension de l'infrastructure portuaire Port-La Nouvelle (Aude). 